BNL-NT-04/17

\title{
Free energy of a static quark anti-quark pair and the renormalized Polyakov loop in three flavor QCD
}

\author{
P. Petreczky and K. Petrov \\ Physics Department, Brookhaven National Laboratory, Upton NY 11973
}

\begin{abstract}
We study the free energy of a static quark anti-quark $(Q \bar{Q})$ pair at finite temperature in three flavor QCD with degenerate quark masses using $N_{\tau}=4$ and 6 lattices with Asqtad staggered fermion action. The static free energy was calculated for different values of the quark mass and the entropy contribution at large distances has been extracted. We also calculate the renormalized Polyakov loop following the approach by Kaczmarek et al.
\end{abstract}

\section{INTRODUCTION}

Non-perturbatively the in-medium modification of inter-quark forces, e.g. screening of fundamental charges at finite temperature, is usually studied in terms of the free energy of static (anti)quarks 1, 2, 3, 4, [5, 6, 7]. More precisely one calculates the difference in the free energy of the system with static quarks and the same system without quarks at fixed temperature. The free energy of static quark anti-quark for different color channels (singlet, octet and averaged) has been studied in great detail in quenched QCD (pure SU(3) gauge theory) 1, 2, 4] as well as in SU(2) gauge theory $[3,[5]$. In the case of full QCD only the color averaged free energy was extensively studied; the first results for singlet and octet free energy for two flavor QCD have appeared only very recently []. In this paper we are going to study the free energy of static quark anti-quark pair in 3 flavor QCD using the so-called Asqtad staggered fermion action 8] with two different lattice spacings (corresponding to $N_{t}=4$ and 6) at three different quark masses.

Apart from the in-medium modification of inter-quark forces the study of the static free energy is interesting as it can be used to define the renormalized Polyakov loop. Although the Polyakov loop is not an order parameter in the presence of dynamical quarks it shows rapid variation in the transition region and therefore is widely used to describe the transition (crossover) in full QCD. In particular, they are useful for constructing effective mean-field theories 9] and studying the interplay between the chiral and deconfining aspects of the transition [10]. We will study the temperature dependence of the renormalized Polyakov loop which shares most of the properties of the usual Polyakov loop but has a meaningful continuum limit.

The rest of the paper is organized as follows. In section II we discuss the lattice setup, parameters of simulations and the zero temperature potential which turns out to be crucial for the analysis of the finite temperature free energies presented in section III. Conclusions are presented in section IV.

\section{PARAMETERS OF SIMULATIONS AND THE STATIC POTENTIAL AT ZERO TEMPERATURE}

In this section we are going to describe the lattice parameters for which our analysis have been performed. In our study we use staggered fermions with Asqtad action [8]. Our analysis to a large extent is based on the gauge configurations generated by the MILC collaboration using the Asqtad action. Therefore we adopt their strategy for fixing the parameters which is described in Refs. 11, 12, 13], namely the strange quark mass was fixed by requiring that $m_{\eta} / m_{\phi}=0.673$, and the temperature scale (the inverse lattice spacing) was fixed from the scale $r_{1}$ defined from the zero temperature static potential as

$$
\left(r^{2} \frac{d V(r)}{d r}\right)_{r=r_{1}}=1
$$

The RG inspired Ansatz for the gauge coupling dependence of the lattice spacing [14] was used for the scale setting. We use the most recent value of $r_{1}$ extrapolated to continuum and to the physical value of the light quark masses $r_{1}=0.317 \mathrm{fm}[15$. to convert the lattice units to physical units. In Table $\llbracket$ we summarize the lattices, quark masses and the corresponding temperature range used in our analysis.

The free energy of a static quark anti-quark pair contains a lattice spacing dependent divergent piece and thus needs to be renormalized. This can be done by normalizing it to the zero temperature potential at short distances where the temperature dependence of the free energy can be neglected 4]. The static quark potential has been studied by the MILC collaboration at three different lattice spacings and various quark masses [13, 15, 16, 17]. The zero temperature potential is also defined only up to some constant which needs to be fixed. As temperature is varied by varying the lattice spacing we need to specify the form of the zero temperature potential which is valid for the whole 

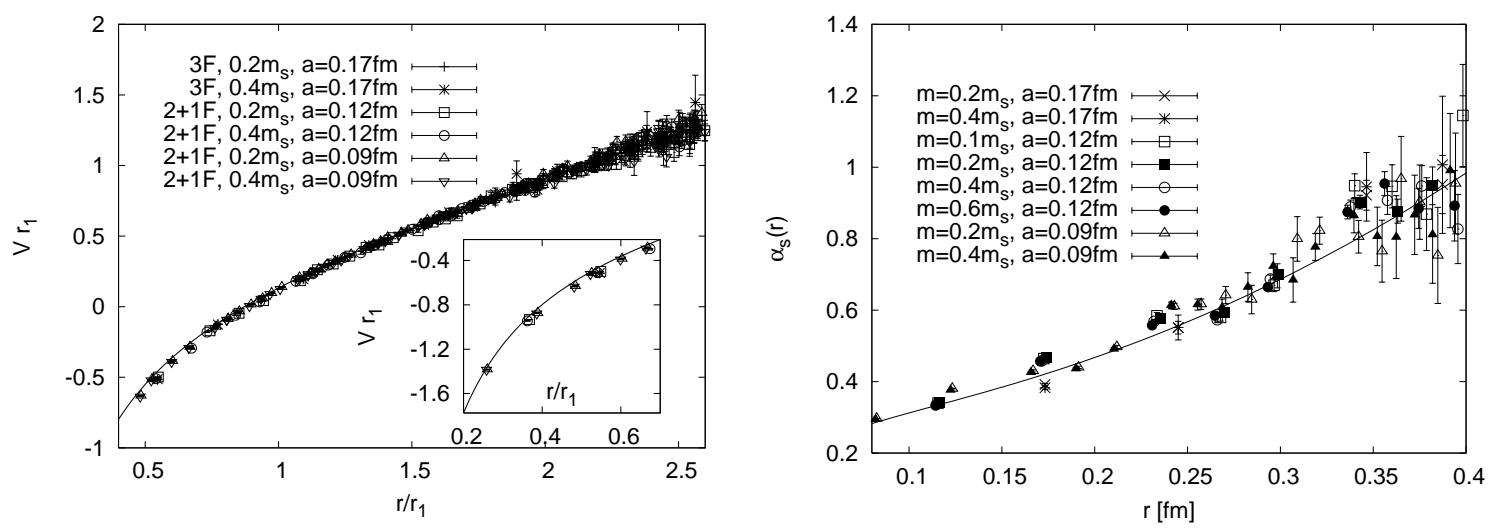

FIG. 1: The static potential at zero temperature from the MILC collaboration versus the parameterization (2) (right) and the effective coupling constant $\alpha_{s}(r)$ as a function of distance (left). Both cases of three degenerate quarks denoted as $3 \mathrm{~F}$ as well as one heavy $\left(m_{q}=m_{s}\right)$ and two light denoted as $2+1 \mathrm{~F}$ are shown.

\begin{tabular}{|l|l|l|}
\hline lattice & $m_{\text {light }}$ & $T$ \\
\hline $12^{3} \times 4$ & $0.2 m_{s}, 0.4 m_{s}, 0.6 m_{s}$ & $135-393 \mathrm{MeV}$ \\
\hline $8^{3} \times 4$ & $0.2 m_{s}, 0.4 m_{s}, 0.6 m_{s}$ & $135-412 \mathrm{MeV}$ \\
\hline $12^{3} \times 6$ & $0.2 m_{s}, 0.4 m_{s}, 0.6 m_{s}$ & $145-310 \mathrm{MeV}$ \\
\hline
\end{tabular}

TABLE I: The parameters of simulations, $m_{s}$ denotes the strange quark mass.

range of lattice spacing relevant in this study, i.e. $0.09 \mathrm{fm}<a<0.3 \mathrm{fm}$. We choose the following form of the zero temperature potential

$$
r_{1} V(x)=-\frac{0.44}{x}+0.56 \cdot x+\frac{0.0125}{x^{2}}, x=r / r_{1}
$$

In Fig. 1 we show this parametrization of the potential against lattice data at three different lattice spacing, and different quark masses. Although in the present paper we discuss the case of three degenerate flavors, we show the zero temperature potential also for $2+1$ flavor case as here more detailed data are available. Because of the very weak quark mass dependence the case of $2+1$ flavors can be used as a good reference. As one can see this parameterization gives a fare description of the data for all lattice spacings and quark masses [17]. The last term in Eq. (2) mimics the effect of the running coupling. In Fig. 1 we also show the effective coupling constant $\alpha_{s}(r)$ defined as

$$
\alpha_{s}(r)=\frac{3}{4} r^{2} \frac{d V(r)}{d r}
$$

One can see again that Ansatz (2) gives a good description of the lattice data. We note that in order to reduce the effects of the breaking of the rotational symmetry in the heavy quark potential following Refs. [4, 18] the separation $r$ between the static charges was redefined as $r \equiv r_{I}=\left(4 \pi C_{L}(r)\right)^{-1}$, where $C_{L}(r)$ is the lattice Coulomb potential for the Luescher-Weisz action [19]. In what follows $r$ will always refer to the modified separation defined above.

\section{THE FREE ENERGY OF STATIC QUARK ANTI-QUARK}

Following Refs. 20, 21] the free energy of static quark-antiquark $(Q \bar{Q})$ pair, i.e. the free energy difference of the system with and without static sources at fixed temperature $T$ in the color singlet and octet channels is defined as

$$
\begin{aligned}
& \exp \left(-F_{1}(r, T) / T+C\right)=\frac{1}{3} \operatorname{Tr}\left\langle W(\vec{r}) W^{\dagger}(0)\right\rangle \\
& \exp \left(-F_{8}(r, T) / T+C\right)=\frac{1}{8}\left\langle\operatorname{Tr} W(\vec{r}) \operatorname{Tr} W^{\dagger}(0)\right\rangle-\frac{1}{24} \operatorname{Tr}\left\langle W(\vec{r}) W^{\dagger}(0)\right\rangle
\end{aligned}
$$

Here $W(\vec{x})=\prod_{\tau=0}^{N_{\tau}-1} U_{0}(\tau, \vec{x})$ is the temporal Wilson line and $L(\vec{x})=\operatorname{Tr} W(\vec{x})$ is known as the Polyakov loop. As $W(\vec{x})$ is not gauge invariant one needs to fix a gauge in order to calculate $F_{1}$ and $F_{8}$. In this study we use the 

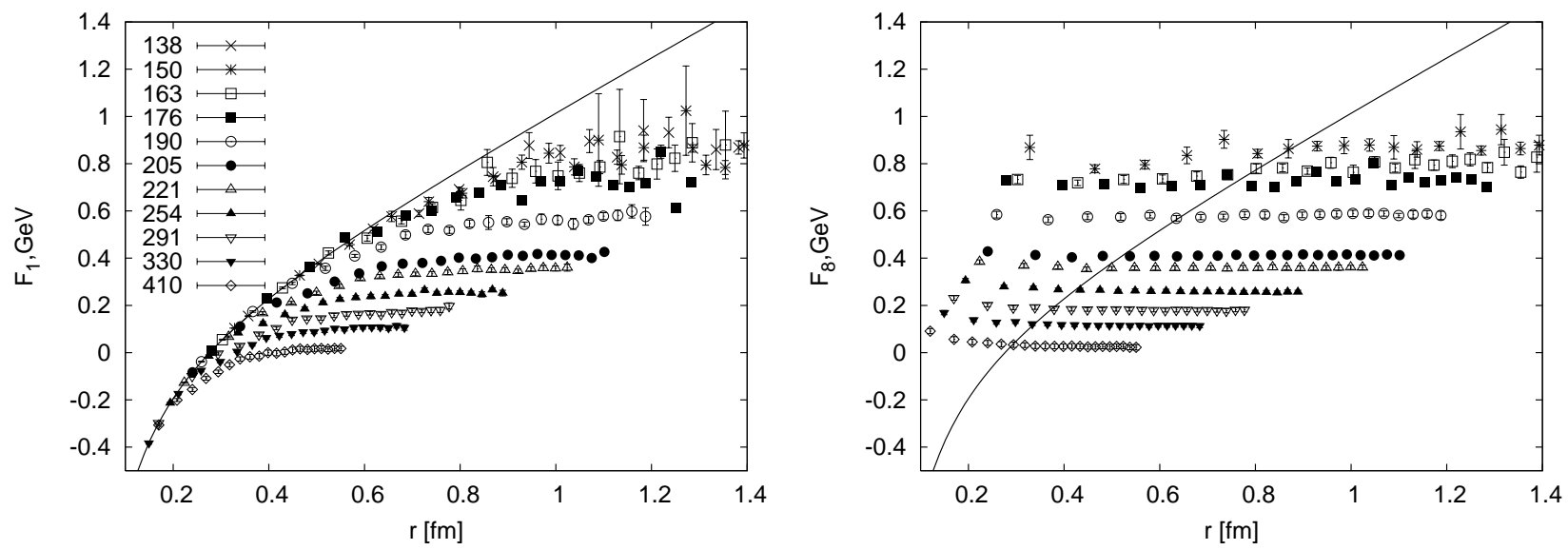

FIG. 2: The singlet (left) and octet (right) free energy of static $Q \bar{Q}$ pair calculated on $12^{3} \times 4$ lattice at $m_{q}=0.4 m_{s}$ at different temperature in $\mathrm{MeV}$. The solid line is the parameterization of the zero temperature potential by Eq. 2

Coulomb gauge as for this gauge a transfer matrix can be constructed and in the zero temperature limit the usual static potential will be recovered. Alternatively one can replace the Wilson line $W(\vec{x})$ by a dressed gauge invariant Wilson line $\tilde{W}(\vec{x})$ using the eigenvectors of covariant spatial Laplacian [3]. The dressed Wilson line, however, is a non-local operator. Both definitions turned out to equivalent [3] (at least numerically). One can also consider the color averaged free energy defined as

$$
\exp \left(-F_{a v}(r, T) / T+C\right)=\frac{1}{9}\left\langle\operatorname{Tr} W(\vec{r}) \operatorname{Tr} W^{\dagger}(0)\right\rangle \equiv \frac{1}{9}\left\langle L(\vec{r}) L^{\dagger}(0)\right\rangle
$$

which can be written as a thermal average of the singlet and octet free energies

$$
\exp \left(-F_{a v}(r, T) / T\right)=\frac{1}{9} \exp \left(-F_{1}(r, T) / T\right)+\frac{8}{9} \exp \left(-F_{8}(r, T) / T\right) .
$$

This quantity is expressed in terms of local explicitly gauge invariant operators and for this reason was extensively studied in the past also in full QCD 22, 23]. The normalization constant $C$ needs to be fixed using some physical normalization condition. As we expect that at very short distances the free energy of static quark anti-quark pair is only given by their interaction energy in the vacuum we fix $C$ by requiring that the singlet free energy coincides with the zero temperature potential given by Eq. (2).

We start the discussion of our numerical results with the case of the quark of mass $0.4 m_{s}$ on the $12^{3} \times 4$ lattice [27]. The corresponding numerical results for the singlet and octet free energy are shown in Fig. 2 The singlet free energy approaches a finite value at large distances which is usually interpreted as string breaking at low temperature and screening at high ones. Note that the distance where the free energy effectively flattens is temperature dependent; it becomes smaller at higher temperatures. At small distances the singlet free energy is temperature independent and coincides with the zero temperature potential. This is intuitively expected as at small distances medium effects are not important. Similar observation has been made for quenched QCD [4, 6, 7]. The octet free energy shows much stronger temperature dependence. At short distances it is expected to have a repulsive tail, which is clearly visible at high temperatures. At low temperatures the presence of such a repulsive tail is less obvious. The reason for this is the following. At low temperatures no data is available at really short distances $(<0.2 \mathrm{fm})$ and the (large distance ) asymptotic value of the octet free energy is large compared to the value of the repulsive tail (which naively is $\alpha_{s} /(6 r)$ ). Also the statistical accuracy is lowest at low temperatures. As the temperature increases both the value of the free energy at large distances is smaller and the statistical accuracy is higher; in addition more data at shorter distances become accessible. The numerical results for other values of the quark masses are similar.

As $N_{\tau}=4$ lattices correspond to a quite large lattice spacing, one may worry about lattice artifacts in the static free energies. Therefore we have compared the singlet free energies calculated from $N_{\tau}=4$ and $N_{\tau}=6$ lattices at approximately same temperatures as shown in Fig. [3 As one can see, there is no sizeable lattice spacing dependence even at the lowest temperatures where the lattice spacing is the largest. Thus calculations of the quark anti-quark free energy on $N_{\tau}=4$ lattices are justified.

We also investigate the temperature dependence of the color averaged free energy. In general due to the presence of color octet contribution the temperature dependence of the color averaged free energy is stronger than that of the 

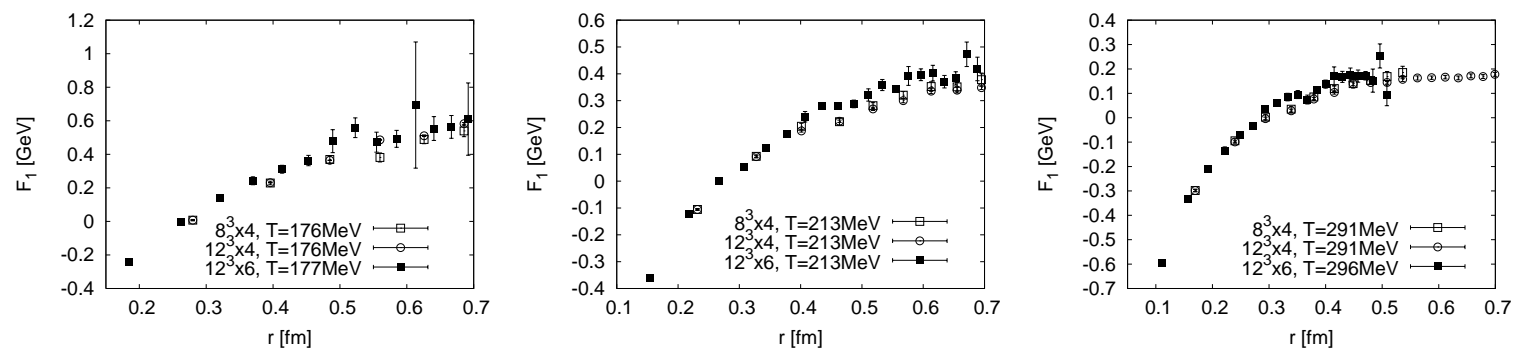

FIG. 3: The singlet free energy calculated on $8^{3} \times 4,12^{3} \times 4$ and $12^{3} \times 6$ lattices at three different temperatures.

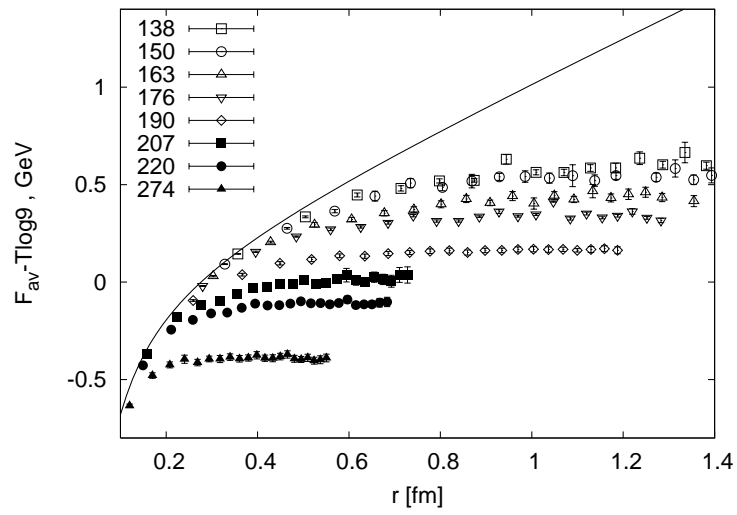

FIG. 4: The color averaged free energy at different temperatures calculated on $N_{\tau}=4$ (open symbols) and $N_{\tau}=6$ (filled symbols) lattices. The solid line is the zero temperature potential.

singlet free energy [23]. At small distances, however, it is expected to be dominated by color singlet contribution and the approximate relation $F_{a v}(r, T) \simeq F_{1}(r, T)+T \ln 9$ should hold [4]. Therefore in Fig. 目 we show $F_{a v}(r, T)-T \ln 9$ at different temperatures including results both from $N_{\tau}=4$ and 6 lattices. As one can see from the figure for temperatures $T<274 \mathrm{MeV} F_{a v}-T \ln 9$ coincides with the zero temperature potential at the shortest distance available in this study. As at the shortest distance the zero temperature potential coincides with the singlet free energy (due to normalization) this in turn implies that the relation $F_{a v}=F_{1}+T \ln 9$ holds at this distance.

As expected, the temperature dependence of the color averaged free energy is stronger than of the color singlet one.

From Figs 24 we see that the free energy for all color channels reach a constant value at large distances $F_{\infty}^{i}(T)=$ $\lim _{r \rightarrow \infty} F_{i}(r, T), \quad i=1,8, a v$. In the infinite volume limit we expect that $F_{\infty}^{1}(T)=F_{\infty}^{8}(T)=F_{\infty}^{a v}(T)$ as at very large distances the free energy of a static quark and anti-quark should not depend on their relative color orientation. This is in fact confirmed in the deconfined phase of pure gauge theory [4, 5, 6]. On our small lattices, $8^{3} \times 4,12^{3} \times 6$ we see small differences between $F_{\infty}^{1}$ and $F_{\infty}^{8, a v}$ which vanish within statistical errors when we go to $12^{3} \times 4$ lattice. In practice we determine $F_{\infty}^{i}(T)$ by fitting the corresponding free energies by a constant at large distances. The lower limit of the fit range was always chosen such that the determined value of $F_{\infty}^{i}$ does not depend on it within our statistical accuracy.

The Polyakov loop $L(x)=\operatorname{Tr} W(x)$ is the order parameter for deconfining transition in pure gauge theories. In full QCD, where dynamical quarks are present, it is no longer an order parameter as it has non-zero value in low temperature phase. Nevertheless, it is used to study the deconfining aspects of the transition in full QCD as well in effective models [9, 10]. The correlator of Polyakov loops corresponding to the color averaged free energy satisfies the cluster decomposition

$$
\left.\left\langle L(r) L^{\dagger}(0)\right\rangle\right|_{r \rightarrow \infty}=|\langle L(0)\rangle|^{2} .
$$

Therefore following Ref. [4], we can define the renormalized Polyakov loop as

$$
L_{r e n}=\exp \left(-\frac{F_{\infty}^{a v}(T)}{2 T}\right) .
$$

The numerical results for $L_{r e n}(T)$ will be discussed in detail at the end of this section. In three flavor QCD for 

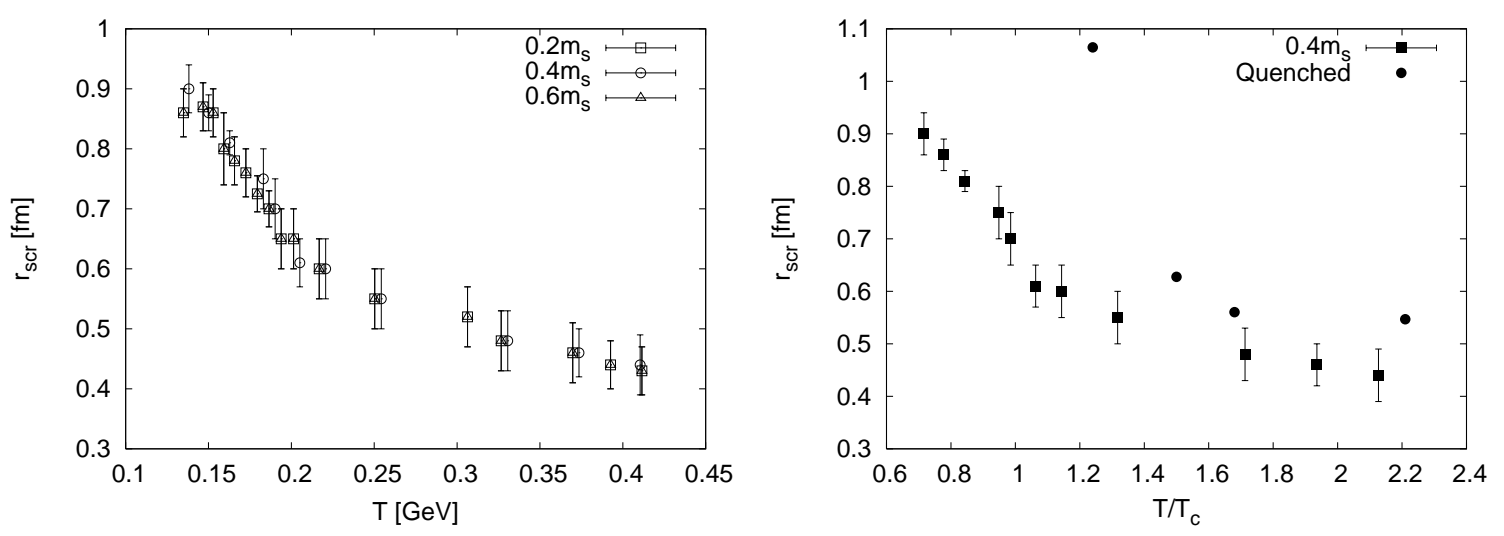

FIG. 5: The effective screening radius from the singlet free energy as a function of the temperature for three different quark masses (left) and as function of $T / T_{c}$ for $0.4 m_{s}$ and quenched QCD (right)

the quark masses used in this study there is no phase transition but only a crossover [11, 12, 13, 24]. Nevertheless, both the chiral condensate and the unrenormalized Polyakov loop show a rapid change at approximately the same temperature 11, 12, 13, 23 referred to as the transition temperature $T_{c}$. Therefore we can define the transition temperature as the temperature where $\partial L_{r e n}(T) / \partial T$ has a maximum, or equivalently $\partial F_{r e n}(T) / \partial T$ has a minimum. We have determined $T_{c}$ in this way and have found it to be consistent with the value of $T_{c}$ defined from the peak of the chiral susceptibility [11, 12, 13]. In the following discussion we will find instructive to plot different quantities as function of $T / T_{c}$, especially when comparing with calculations done in $\mathrm{SU}(3)$ gauge theory.

To characterize the range of interaction in the medium it is convenient to introduce the effective screening radius $r_{s c r}$. It is defined as the distance at which the singlet free energy is only $10 \%$ below its asymptotic value $F_{1}(r=$ $\left.r_{s c r}, T\right)=0.9 F_{\infty}^{1}(T)$. Here $F_{\infty}^{1}(T)$ is the asymptotic value of the singlet free energy at infinite separation. In Fig. [5 we show the values of $r_{s c r}$ for three different quark masses and $12^{3} \times 4$ lattices. Certainly as $F_{1}(r, T)$ has statistical errors, it is difficult to determine exactly at which distance $r$ the equation $F_{1}\left(r=r_{s c r}, T\right)=0.9 F_{\infty}^{1}(T)$ holds. We have tried to estimate this uncertainties in the values of $r_{s c r}$ and show them in Fig. 5 as errors bars. At small temperatures the value of the screening radius is about $0.9 \mathrm{fm}$ and is temperature independent. As we increase the temperature $r_{s c r}$ decreases reaching the value of $0.5 \mathrm{fm}$ at the highest temperature. Note that the temperature dependence of $r_{s c r}$ is roughly the same for all quark masses. In Fig. [5] we also show the effective screening radius for $0.4 m_{s}$ and quenched QCD estimated from the singlet free energies of Ref. [4] as function of $T / T_{c}$. Close to $T_{c}$ the screening radius in quenched QCD is considerably higher than in three flavor QCD but at higher temperatures they are comparable.

Now we are going to investigate the temperature and quark mass dependence of the asymptotic value of the free energy and identify the entropy contribution to it. In the following discussion we will take the asymptotic value of the free energy in the infinite volume limit to be given by the color averaged value $F_{\infty}(T) \equiv F_{\infty}^{a v}(T)$ and also skip the index $i$ in the following discussion. The numerical results for $F_{\infty}(T)$ at three different quark masses are shown in Fig. [6] One immediately notices that the quark mass dependence may vanish at small temperatures $(T<150 \mathrm{MeV})$ and definitely negligible at high temperatures $(T>250 \mathrm{MeV})$ while large mass dependence is observed in the transition region. One may wonder to which extent this mass dependence is due to the mass dependence of the transition temperature $T_{c}$. Therefore in Fig. [6] we also show the data for $F_{\infty}(T)$ versus $T / T_{c}$ which shows that up to $T / T_{c}=1$ there is no mass dependence. This is in accordance with finding of Ref. 23] where for quark masses below the strange quark mass the mass dependence of $F_{\infty}$ is quite small for $T / T_{c}$ close to one. Thus for temperatures below $T_{c}$ the mass dependence of $F_{\infty}(T)$ can be understood in terms of mass dependence of $T_{c}$. However, as one can also see from Fig. [6] for $T / T_{c}>1$ substantial mass dependence is seen.

In the case of very small temperatures we expect $F_{\infty}(T)$ to be temperature independent and related to twice the binding energy of a heavy-light $(D-$ or $B-)$ meson $2 E_{b i n}=2 M_{D, B}-2 m_{c, b}$. More precisely, it should be the binding energy of a static-light system as found in the 3d SU(2) Higgs model which is quite similar to QCD [25]. Because of the heavy quark symmetry we expect the binding energy of a heavy-light meson to be the same as of the static-light system. Based on this observation it has been argued in Ref. [26] that the decrease of $F_{\infty}(T)$ with the temperature close to $T_{c}$ implies the decrease of the $M_{D, B}$ leading to quarkonium suppression. However, one should keep in mind that $F_{\infty}(T)$ also contains an entropy contribution. The entropy due to the presence of a static $Q \bar{Q}$ pair can be calculated as

$$
S_{\infty}(T)=-\frac{\partial F_{\infty}(T)}{\partial T}
$$



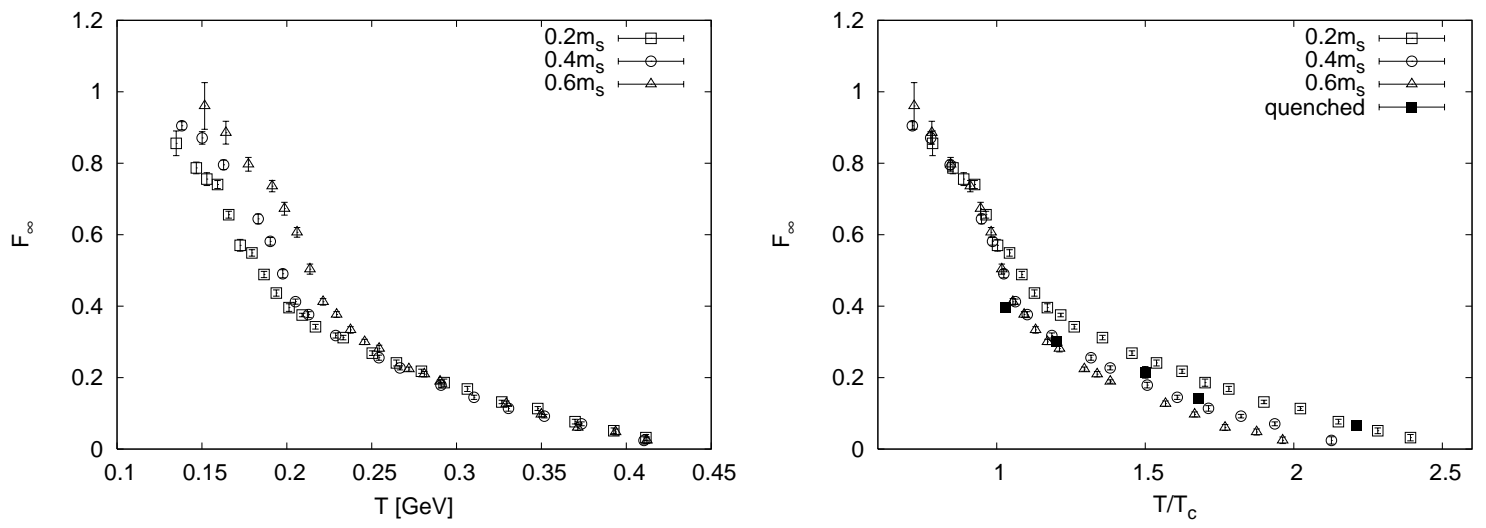

FIG. 6: The asymptotic value of the free energy at large distance for different quark masses as function of the temperature in physical units(left) and of $T / T_{c}$ (right). On the right plot we also show the value of $F_{\infty}$ from pure gauge theory [4, 6].
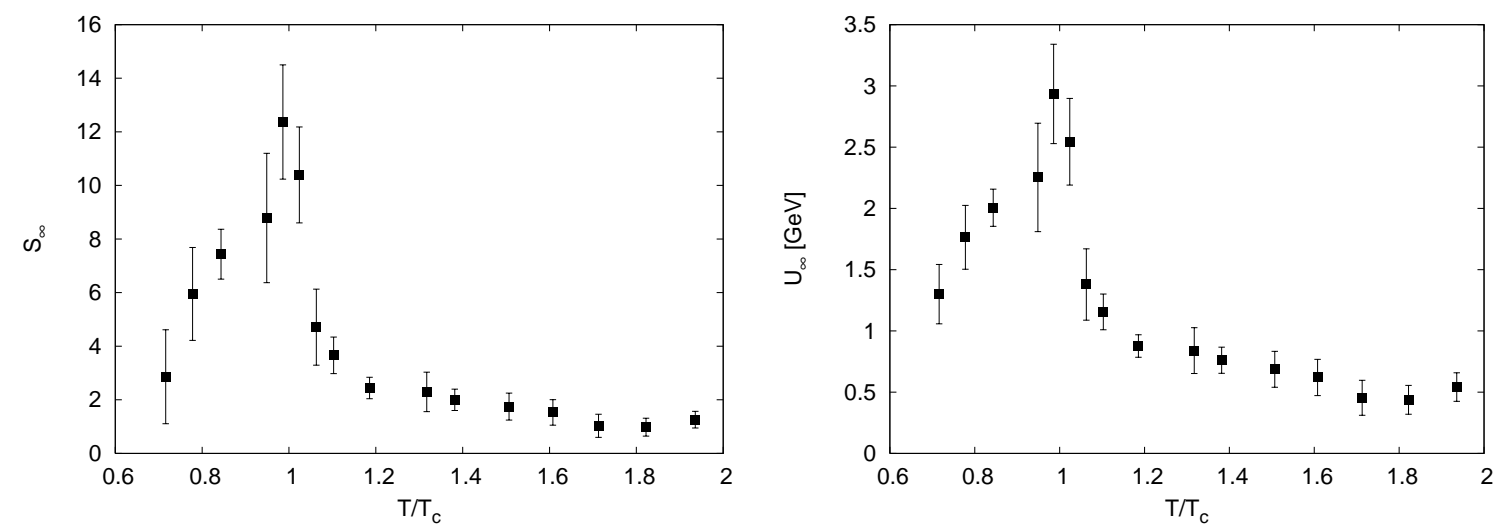

FIG. 7: The asymptotic values of the entropy and the internal energy of static $Q \bar{Q}$ pair at large distances as function of the temperature.

We also can calculate the energy induced by a static $Q \bar{Q}$ pair $U_{\infty}=F_{\infty}+T S_{\infty}$. Numerically the derivative with respect to the temperature in Eq. (10) was estimated using forward differences. In Fig. 7 we show the entropy $S_{\infty}$ and energy $U_{\infty}$ as functions of temperature. Both the entropy and the energy show a strong increase near $T_{c}$ and with our definition of $T_{c}$ peak exactly at $T_{c}$. This large increase in entropy and energy is probably due to many-body effects and makes the interpretation of $U_{\infty}$ as the binding energy of heavy-light meson not very plausible.

The numerical results for $F_{\infty}(T)$ can be easily converted to the numerical results for the renormalized Polyakov loop $L_{r e n}(T)$ which are shown in Fig. 8 The mass dependence of $L_{r e n}(T)$ is the same as for $F_{\infty}$, when plotted versus temperature in physical units it shows mass dependence for $150 \mathrm{MeV}<\mathrm{T}<250 \mathrm{MeV}$ and when plotted as function of $T / T_{c}$ mass dependence for $T / T_{c}>1$. The renormalized Polyakov loop should not depend on the lattices spacing. To check this we have calculated $L_{r e n}(T)$ also on $12^{3} \times 6$ and $8^{3} \times 4$ lattices. The results are summarized in Fig. 8 and as one can see the $N_{\tau}$ (lattice spacing) dependence of $L_{r e n}(T)$ is quite small.

\section{CONCLUSIONS}

In this paper we have presented calculations of the free energy of a static quark anti-quark pair in three flavor QCD for several quark masses. We have found that the free energy gets screened beyond some distance for all temperatures, as expected. For small temperature this distance, the effective screening radius, does not depend on the temperature and is about $0.9 \mathrm{fm}$. As the temperature increases the effective screening radius decreases. Using the asymptotic value of the free energy we have defined the renormalized Polyakov loop and proved its scaling with the lattice spacing. We have also identified the entropy contribution to the free energy as well as the internal energy at large distances and found that they show strong increase at $T_{c}$. We have found substantial quark mass dependence in the vicinity of the transition. In the future it will be certainly interesting to extend this study to larger lattices and to study the 

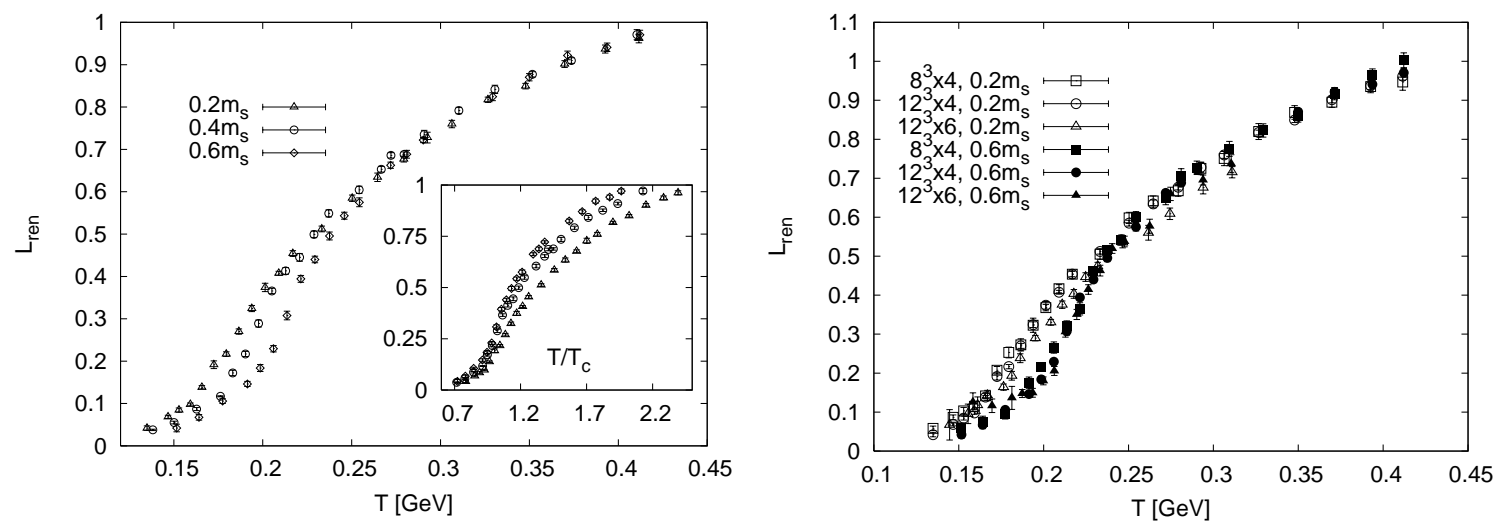

FIG. 8: The renormalized Polyakov loop calculated on $12^{3} \times 4$ lattices for three different quark masses (left) and for $m_{q}=$ $0.2 m_{s}, 0.6 m_{s}$ on $8^{3} \times 4,12^{3} \times 4$ and $12^{3} \times 6$ lattices (right). The insertion in the left figure shows the renormalized Polyakov loop as function of $T / T_{c}$ (see text). .

dependence of the entropy contribution as function of the separation which because of the limited statistics was not possible here. Many properties of the static quark anti-quark free energies presented in this three flavor study turns out to be similar to the preliminary findings in two flavor case by the Bielefeld group reported in Ref. 7].

\section{Acknowledgment}

Most of our analysis has been performed using finite temperature configurations from MILC collaboration. We would like to thank the members of MILC collaboration for sharing these configurations as well as for the unpublished data on the zero temperature potential. Special thanks goes to C. DeTar, U.M. Heller, R. Sugar and D. Touissant for many helpful communication concerning their results on zero temperature potential, scale setting and other issues. We would like to thank F. Karsch and A. Patkós for careful reading of the manuscript and several valuable suggestions. This work has been supported by the US Department of Energy under the contract DE-AC02-98CH10886 and by the SciDAC project.

[1] N. Attig et al., Phys. Lett. B209, 65 (1988)

[2] O. Kaczmarek, F. Karsch, E. Laermann, M. Lütgemeier, Phys. Rev. D62, 034021, (2000)

[3] O. Philipsen, Phys. Lett. B535, 138 (2002)

[4] O. Kaczmarek, F. Karsch, P. Petreczky and F. Zantow, Phys. Lett. B543, 41 (2002)

[5] S. Digal, S. Fortunato and P. Petreczky, Phys. Rev. D68, 034008 (2003)

[6] O. Kaczmarek, F. Karsch, P. Petreczky and F. Zantow, Nucl. Phys. B (Proc. Suppl.) 129-130, 745 (2004), hep-lat/0309121

[7] O. Kaczmarek et al., hep-lat/0312015

[8] T. Blum et al, Phys. Rev. D55, 1133 (1997); J. F. Lagae and D.K. Sinclair, Phys. Rev. D59, 014511 (1999); K. Orginos, D. Toussaint and R.L. Sugar, Phys. Rev. D60, 054503 (1999)

[9] A. Dumitru and R.D. Pisarski, Phys.Lett. B525, 95 (2002); Phys.Rev. D66 (2002) 096003; A. Dumitru, D. Röder, J. Ruppert, hep-ph/0311119 A. Dumitru, Y. Hatta, J. Lenaghan, K. Orginos, R.D. Pisarski, hep-th/0311223

[10] A. Mócsy, F. Sannino, K. Touminen, Phys. Rev. Lett. 92, 182302 (2004), hep-ph/0403160 hep-ph/0401149

11] C. Bernard et al. [MILC Collaboration], Nucl. Phys. B (Proc. Suppl.) 129-130, 626 (2004), hep-lat/0309118

[12] C. Bernard et al. [MILC Collaboration], Nucl. Phys. B ( Proc. Suppl.) 119, 523 (2003), hep-lat/0209079

[13] C. Bernard et al. [MILC Collaboration], Nucl. Phys. A702, 140 (2002)

[14] C.R. Alton, Nucl. Phys. B (Proc. Suppl.) 53, 867 (1997), hep-lat/9610016

[15] C. Aubin et al., hep-lat/0402030

[16] C. Bernard et al. [MILC Collaboration],Phys.Rev. D62, 034503 (2000)

[17] unpublished data on zero temperature potential from MILC collaboration, private communications with U.M. Heller and D. Toussaint

[18] S. Necco and R. Sommer, Nucl.Phys. B622, 328 (2002)

[19] P. Weisz, Nucl. Phys. B212, 1 (1983); P. Weisz and R. Wohlert, Nucl. Phys. B236, 397 (1984); ibid. B247, 544 (1984)

[20] L.D. McLerran and B. Svetitsky, Phys. Rev. D24, 450 (1981) 
[21] S. Nadkarni, Phys. Rev. D33, 3738 (1986)

[22] C. DeTar, O. Kaczmarek, F. Karsch and E. Laermann, Phys. Rev. D59, 031501 (1999)

[23] F. Karsch, E. Laermann and A. Peikert, Nucl. Phys. B605, 579 (2001)

[24] F. Karsch, E. Laermann, C. Schmidt, Phys.Lett. B520, 41 (2001)

[25] O. Philipsen and H. Wittig, Phys. Rev. Lett. 81, 4056 (1998)

[26] S. Digal, P. Petreczky and H.Satz, Phys. Lett. B514, 57 (2001)

[27] Most of the configurations used for our measurements were provided to us by the MILC collaboration. 